\title{
Answer to Medical Quiz: Images
}

1. T2, FLAIR, DWI weighted magnetic resonance scan image showing bilaterally symmetrical hyperintensities in Caudate nucleus, Putamen, with sparing of Globus Pallidus, suggestive of Extrapontine myelinolysis.(Fig-A,B,C)

T2-weighted image showing hyperintensity in the pons with sparing of the peripheral fibers suggestive of pontine myelinolysis (Fig: D)

2. Osmotic Demyelination Syndrome

3. Rapid correction of sodium

4. Multiple sclerosis, Brainstem \& basal ganglia infarction

\section{Osmotic demyelination syndrome}

Central pontine myelinolysis (CPM), also known as Osmotic demyelination syndrome or Central pontine demyelination, is a neurological disease caused by severe damage of the myelin sheath of nerve cells in the brainstem, more precisely in the area termed the pons, predominately of iatrogenic etiology. It is characterized by acute paralysis, dysphagia (difficulty swallowing), and dysarthria (difficulty speaking), and other neurological symptoms. It can also occur outside the pons. ${ }^{1}$ The term "osmotic demyelination syndrome" is similar to "central pontine myelinolysis", but also includes areas outside the pons. ${ }^{2}$

Central pontine myelinolysis presents most commonly as a complication of treatment of patients with profound, life-threatening hyponatremia (low sodium). It occurs as a consequence of a rapid rise in serum tonicity following treatment in individuals with chronic, severe hyponatremia who have made intracellular adaptations to the prevailing hypotonicity. Hyponatremia should be corrected at a rate of no more than $8-12 \mathrm{mmol} / \mathrm{L}$ of sodium per day to prevent central pontine myelinolysis. ${ }^{3}$

\section{References}

1. Gocht A, Colmant HJ. Central pontine and extrapontine myelinolysis: a report of 58 cases. Clin Neuropathology 1987; 6 (6): 262-70.

2. Lampl C, Yazdi K. Central pontine myelinolysis. Eur Neurol 2002: 47 (1): 3-10.

3. Babar, S. SIADH Associated With Ciprofloxacin. Annals of Pharmacotherapy (Sage Publishing) 2013: 47 (10): 1359-63. 


\section{Medical Quiz: SBA - Answers}

\section{Question No. 1: Correct Answer -A}

The case in this question describes the presentation of a patient with shingles. This is caused by reactivation of VZV. The normal presentation of shingles is with a prodromal phase of tingling or pain, followed by an eruption of a painful, blistering rash in a dermatomal distribution. Complications of shingles include postherpetic neuralgia (severe pain in the dermatomes affected after shingles) and ocular disease. Early in the disease, as in this case, oral aciclovir is the most appropriate treatment as it may help shorten the course of the disease. High-dose intravenous acyclovir is given to immunocompromised patients. Topical steroids are not helpful in the management of shingles. Paracetamol is an important analgesic for symptomatic relief of patients with shingles, but in early onset disease acyclovir should be given to try and reduce the course of the disease, making this the incorrect answer. Amitryptilineis a useful drug for the management of post-herpetic neuralgia, but is not used for the management of shingles.

\section{Question No. 2: Correct Answer - D}

Dacrocytes, more commonly known as tear-drop cells, are strongly indicative of myelofibrosis. Sickle cells occur due to homozygous haemoglobin $\mathrm{S}$ which causes cell sickling on polymerization. Schistocytes, more commonly known as fragmented red cells, can be seen in a number of conditions such as haemolyticanaemia or disseminated intracascular coagulation. Spherocytes are commonly seen in haemolyticanaemia or in congenital disease, such as hereditary spherocytosis. Target cells can be indicative of obstructive jaundice, liver disease, haemoglobinopathies and hyposplenism.

\section{Question No. 3: Correct Answer -A}

Psoriasis, one of the most common dermatological presentations, is a chronic inflammatory skin condition characterized by the presence of well-defined, erythematous plaques with silvery scales, usually present on the extensor surfaces of the elbows, knees, scalp and sacrum. Plaques, without silvery scales, may be present on flexures (e.g. axillae, groin). Associated signs, which may be present, include nail changes, Kobner phenomenon (lesions appearing along sites of traumatic skin injury), Auspitz sign (pinpoint bleeding when scale removed) and systemic signs such as arthropathy (e.g. asymmetrical oligomonoarthritis, arthropathy of the distal interphalangeal joints (DIPs), rheumatoid-like sero-negative polyarthritis, arthritis mutilans and psoriatic spondylitis). Management is targeted at disease control and includes- education, stress and alcohol avoidance, topical drugs are regarded as the mainstay of therapy (e.g. tar, dithranol, vitamin D analogues, topical steroid and antibiotic/fungal treatment is beneficial in flexural disease). For psoriasis that is not responsive to conventional therapy (also known as relcalcitrant psoriasis), topical retinoids, phototherapy, oral drugs (e.g. methotrexate, cyclosporin, acitretin, hydroxycarbamide) may be considered. Parenteral cytokine inhibitors or monoclonal antibodies may be considered for recalcitrant psoriasis unresponsive to systemic therapy mentioned above. The question states that the patient is unresponsive to conventional therapy. Therefore, the next step would be to consider topical retinoid therapy.

\section{Question No.4: Correct Answer - E}

Reactive arthritis is a sterile arthritis, which follows an attack of dysentery (caused by Campylobacter, Salmonella, Shigellaor Yersinia spp.) or urethritis (caused by Chlamydia or Ureaplasmaspp.). Clinical features of reactive arthritis are an acute, asymmetrical lower limb arthritis occurring $1-4$ weeks following an infection. Other features of reactive arthritis include conjunctivitis (as described by this case), enthesitis (which may result in plantar fasciitis or Archilles tendonitis), circinate balanitis (painless superficial ulceration of glans penis), keratoderma blenorrhagica (painless, red plaques on the soles and palmes), nail dystrophy, mouth ulcers and, rarely, aortic incompetence. The triad of urethritis, arthritis and conjunctivitis is known as Reiter's disease. Treatment of reactive arthritis is with NSAIDs and local steroid injection for symptomatic control. Any underlying infection should be treated but is unlikely to influence the course of the arthritis. Individuals who develop recurrent attacks of arthritis can be considered for therapy with sulfasalazine or methotrexate. Septic 
arthritis is a monoarthritis, presenting as a hot, swollen, tender joint. Therefore this is the incorrect answer. Gout also usually presents as an acute monoarthritis and does not cause conjunctivitis or urethritis, making this answer incorrect. Ankylosing spondylosis does not affect the knees or cause urethritis, although it can cause conjunctivitis. Enteropathic arthritis is an asymmetrical lower limb arthritis, associated with inflammatory bowel disease. The absence of bowel symptoms in this patient means this is the incorrect answer.

\section{Question No.5: Correct Answer - E}

The patient appears to be suffering from cushingoid symptoms. After a history to exclude causes such as high-dose steroid intake, the main differential diagnoses include an adrenal tumour, an ectopic tumour producing ACTH (Cushing's syndrome) or a pituitary tumour (Cushing's disease). Although a 24-hour urinary free cortisol level measurement does not confirm the exact diagnosis, it does indicate if there is a pathological excess of cortisol (levels can vary up to $700 \mathrm{nmol} / \mathrm{L}$ in the morning to $280 \mathrm{nmol} / \mathrm{L}$ at midnight). A low dose ( $0.5 \mathrm{mg})$ dexamethasone test involves measuring ACTH after dexamethasone administration. In Cushing's disease and syndrome, there is no suppression of ACTH. A high dose dexamethasone test will differentiate between Cushing's disease and Cushing's syndrome since only in the former is there suppressed ACTH production after high-dose dexamethasone administration. A CT scan can be used to identify a pituitary tumour if requiring surgical management. Urinary catecholamine measurement is used in the diagnosis of a phaeochromocytoma, an adrenal tumour producing excess catecholamines measurable in the urine. Differentiating an adrenal tumour producing excess cortisol can be done by administering metyrapone, an 11- $\beta$ hydroxysteroid dehydrogenase inhibitor, which effectively ceases adrenal cortisol production. If cortisol is still high it is due to an ectopic source, e.g. lung tumour. 